\title{
Does Informing Referring Practices About Visit Non-Compliance Improve Subsequent Show Rates to a Pediatric Cardiology Practice?
}

Valerie A. Schroeder, M.D.

University of Kansas Medical Center, Division of Pediatric Cardiology

Kansas City, KS

\begin{abstract}
Background. Missed specialty appointments are common. Consequently, patients may never receive intended sub-specialty care. We predicted that no-show (NS) notification would result in more successful encounters following a NS.

Methods. Referring practices were surveyed regarding how NS communication may change patient management. To test the effect of NS notification, two prospective patient groups were evaluated: a non-notification group (Control) and a NS-notification group (Intervention). Patients were tracked seven months to determine rates and time to a successful encounter. Group differences were assessed by either a two sample Z-test for proportions or an independent t-test.

Results. The survey indicated that $43.7 \%$ of practices routinely receive NS notification from subspecialists. For $69 \%$, NS notification would prompt patient/family contact. Baseline NS rates for the Control group $(n=633)$ was $10 \%(n=67)$ and for the Intervention group $(n=623)$ was $13.5 \%(\mathrm{n}=83, \mathrm{p}=0.1)$. Rates of eventual successful encounters among NS patients were $28 \%$ for the Control group and $11 \%$ for the Intervention group $(p=0.21)$. Mean time to successful encounter was shorter in the Intervention group (Control, 2.9 months $+/-2$; Intervention, 1.65 months $+/-0.9, \mathrm{p}=0.045$ ).

Conclusion. Unlike adult studies, pediatric practitioners likely would intervene if a NS was known. Although fewer patients were seen in the NS notification group, the time to encounter was shorter for the Intervention group compared to Controls. While NS notification may not lead to more successful encounters, enhanced communication to the referring practice may ensure that the most worrisome patients are seen promptly.
\end{abstract}

KS J Med 2013; 6(2):51-59.

\section{Introduction}

Missed medical appointments are common. Failure to keep appointments may decrease office productivity and impact patient health. ${ }^{1,2}$ Visit non-compliance rates vary ranging from $4-15 \%$ in primary care, ${ }^{3}$ $31-40 \%$ in pediatric subspecialty clinics, ${ }^{3}$ and $20-30 \%$ for mental health and other adult subspecialties. ${ }^{4}$ Although many successfully re-schedule, up to $50 \%$ of mental health patients drop completely out of scheduled care. ${ }^{4}$ One study tracked nearly 7,000 primary-care patients age 65 or older and discovered that only $50 \%$ of those patients received the intended subspecialty care prescribed by their primary physician as a result of missed appointments.

In many practices, it is routine for the subspecialty office to notify primary care offices of a missed office visit out of concern that the missed appointment may otherwise go unrecognized. As a result of this knowledge, one may anticipate that the referring physician would decide (with or without re-contacting the patient) whether the encounter was still appropriate. However, few studies evaluated how missed appointments are perceived and managed by the primary care office. Interestingly, Lloyd 
et al. ${ }^{6}$ interviewed referring physicians regarding their alleged role in re-contacting adult non-attenders to a psychiatric outpatient clinic. The survey suggested that the majority of respondents did not indicate a perceived responsibility for re-contacting their referred patients. Rather, respondents preferred that referral center reschedule appointments up until such time that they would be discharged because of chronic non-compliance. The authors speculated there was a potential risk for referrals being lost to follow-up and consequently compromising care. To our knowledge, there are no data regarding how pediatric offices manage subspecialty NSs. Thus, we were interested in determining whether pediatric practitioners viewed the responsibility of intervening following a NS differently.

The study goals were twofold. First, a mailed survey to referring centers sought to determine if NS notification was deemed helpful in terms of patient management by the primary practitioner. Second, a prospective cohort study was designed to determine whether NS communication resulted in a greater number of successful subsequent encounters compared to no notification. We speculated that pediatric practitioners would accept an active role in helping children to be seen by the cardiologist as needed if NS information was provided.

\section{Methods}

This study was reviewed by the University of Kansas Medical Center Human Subject Committee (IRB) and designated as a quality improvement project. Thus, informed consent was not required for either patients or survey respondents.

Information collected from referring practitioners. Prior to this project, it was not routine to notify referring offices of a NS to cardiology. To assess pediatric practitioners' perspective regarding how NS notification might influence patient management, a survey was mailed to all known actively referring physicians to the University of Kansas Medical Center Pediatric Cardiology clinic during January and February, 2012. A non-validated, quality improvement survey (Appendix) was designed to understand how offices were notified about NS and whether they would act upon this knowledge. The survey also addressed the controversial issue of cancelling referrals in the face of chronic non-attendance. The survey was sent prior to NS data collection and included:

- whether referral centers routinely notify the office of a NS;

- the preferred method to be informed of a NS;

- what action might be taken if there was a NS notification;

- what would influence the decision to contact a family regarding the NS;

- who would be most influential to keep an appointment; and

- should referrals be cancelled after more than three NSs.

Subjects. For study purposes, a missed appointment was defined as a noncancellation either the day before a morning clinic or within a half-day notice of an afternoon clinic (clinical policy). All patients referred to University of Kansas Pediatric Cardiology (0-18 years of age) over two four-month blocks of time (2/12$10 / 12$ ), whether new or established, or seen at either the University of Kansas based practice or outreach clinics (Salina, Hays, Pittsburg, and Topeka Kansas) were included. Patients were excluded if visits were canceled greater than one-half day prior to the appointment, or there was knowledge of a sudden unexpected illness or emergency preventing the visit.

Study design. To test the effectiveness of NS notification upon rates of a subsequent successful encounter, a prospective, nonrandomized study was conducted involving two patient study groups. The Control group 
consisted of a non-intervention group that was used to establish baseline NS rates, baseline rates of successful subsequent encounters, and time to be seen. The Intervention group included patients where written NS notification was mailed to the referring office within two weeks of the missed visit. For each group, all appointments within two consecutive fourmonth time periods were reviewed for NSs.

The Control group was evaluated first, followed by the Intervention group. For both groups, each NS patient was tracked using practice electronic medical record appointment lists for up to seven months following a NS to detect successful encounters. We chose seven months because our next available appointments were generally less than three months, thus most patients should have been able to reschedule within this time-frame.

As per standard practice, all patients were reminded of the upcoming visit (in writing or by phone) and families were contacted to reschedule a missed appointment. A secondary endpoint included the effect of NS notification on time to successful encounter. Other data collected included the referring diagnosis and whether patients were established or new referrals, or local versus outreach patients.

Statistics. Group differences were assessed by either a two sample, one-tailed Z-test for proportions $(95 \%$ confidence level) or a non-paired, one-tailed t-test for continuous variables. A p-value of less than 0.05 was required for statistical significance. Descriptive data are reported as means +/standard deviation (SD) for continuous data or as percentages for categorical data.

\section{Results}

Referring practice survey regarding noshow notification. Surveys were mailed to 151 actively referring primary practices prior to any patient data collection. A total of $26 \%$ of practices returned a completed survey $(\mathrm{n}=39)$. Results indicated that $43.7 \%$ of practices routinely receive NS notification by subspecialists, $43.5 \%$ learn of the NS at the next visit but are not informed by the subspecialist, and $12.8 \%$ may never be informed by either the family or subspecialist (Table 1). All, but one, of the respondents preferred written NS notification. If aware of the NS, 69\% of respondents would contact the family to investigate as necessary, $15 \%$ would reschedule without calling to investigate, $15 \%$ would let family reschedule, $0.8 \%$ would let referral center reschedule.

When asked about who has primary responsibility for mitigating the missed visit, $10 \%$ of respondents checked both referring center and family. Of the total responses, $43 \%$ believed the referring center should remedy the NS. However, an equal number indicated that the family holds responsibility $(43 \%)$. The remaining indicated that either the care team or family were jointly responsible (14\%) or were not sure (10\%).

A majority indicated that consults should be cancelled for more than three NSs $(67.5 \%)$ whereas the remaining was either opposed to cancellation or unsure. Regarding why a practitioner may or may not initiate family contact once aware of the NS, 69\% would intervene based upon perceived acuity of the patient's complaint or physical finding, $20 \%$ would always call the family, and $11 \%$ would call if time allowed.

Effect of no-show notification on rates of successful encounters. The Control group consisted of 633 total referrals where the NS rate was $10 \%(\mathrm{n}=65$; Table 2$)$. The Intervention group included 623 referrals with a NS rate of $13.5 \%(n=84 ; \mathrm{p}=0.1)$. The distribution of new versus established referrals and local versus outreach patients were similar between groups. For both groups, NS rates were lower at outreach 
Table 1. Summary of physician survey responses.

Current Mode of Notification about a No-Show

By referral center

$\%$ of Responses

At patient follow-up

43.7

Never found out

12.8

Practitioner Response to No-Show Notification by Referring Center

Reschedule appointment

15.0

Call family

69.2

Allow referral center to reschedule

0.8

Allow family to reschedule

15.0

Who holds responsibility for missed visits? *

Referring center

43.0

Referral center

Family

43.0

Not sure

10.0

All

14.0

Opinion of cancelling referrals in setting of chronic non-compliance (> 3 missed visits)

Yes

67.5

No

2.8

Not sure

29.7

What would influence decision to call family following no show notification?

Patient acuity

69.0

Always call

20.8

Available free time

10.2

*Some respondents selected more than one answer.

clinics compared to the university clinic (Control, $\mathrm{p}=0.02$; Intervention, $\mathrm{p}=0.01$ ). Only one subject had more than one NS within the study period (only one NS counted for study purposes).

Reasons for referral are summarized in Table 2. Among those missing cardiology appointments, known or suspected congenital heart disease was the primary referring diagnosis $(32 \%)$ for both groups ( $p$ $=0.5)$. Other common diagnoses included electrocardiogram or rhythm concerns (13$15 \%)$, serious familial heart disease (4.6$7.1 \%$ ), orthostatic instability $(4.7-12 \%)$, or chest pain $(2.4-9 \%)$.

Overall rates of successful encounters following a missed visit was $28 \%$ (19 of 65) for the Control group and 11\% (11 of 84) for the Intervention group $(\mathrm{p}=0.1)$. Among Control patients with known or suspected congenital heart disease, 28\% (12 of 43) were seen eventually whereas only $11 \%$ ( 8 of 73) of Intervention patients were seen (p $=0.08$ ). There were no group differences in subsequent follow-up rates comparing either the university versus outreach practices $(\mathrm{p}=$ 0.2 ) or established versus new referral patients $(\mathrm{p}=0.43$ and $\mathrm{p}=0.52$, respectively). However, mean time to successful encounter was shorter in the Intervention group (1.65 months +/- 0.9) compared to the Control group (2.9 months $+/-2 ; \mathrm{p}=0.045)$. 
Table 2. Characteristics of patients that missed visits.

\begin{tabular}{lccc}
\hline & Control & Intervention & p value \\
Referring Diagnoses (\%) & & & 0.50 \\
Suspected/Known Congenital Disease & 32.0 & 32.0 & 0.50 \\
Non-Congenital Referrals & 68.0 & 68.0 & \\
$\quad$ Chest Pain & 9.0 & 2.4 & \\
$\quad$ Rhythm/EKG & 15.0 & 13.0 & \\
$\quad$ Dizzy/Syncope & 12.0 & 4.7 & \\
$\quad$ Family History & 4.6 & 7.1 & \\
$\quad$ Hypertension & 1.5 & 7.1 & \\
$\quad$ Dyslipidemia & 3.0 & 2.3 & \\
$\quad$ Pulmonary Hypertension & 0.0 & 0.0 & 0.42 \\
$\quad$ Marfan Syndrome & 0.0 & 1.2 & 0.30 \\
$\quad$ Other & 4.6 & 6.0 & 0.60 \\
Referral Demographics (\%) & & & 0.49 \\
Local & 44.6 & 33.4 & \\
Outreach & 55.4 & 66.6 & \\
Established & 41.5 & 43.0 & 0.27 \\
New & 58.5 & 57.0 & 0.08 \\
Successful Encounters following a Missed Visit & & $\mathbf{0 . 0 4 5}$ \\
\% Total Successful encounters & 28.0 & 11.0 & \\
\% Suspected congenital disease seen & 31.0 & 15.0 & \\
Time to encounter (months) & $2.9+/-2$ & $1.65+/-0.9$ & \\
\hline
\end{tabular}

\section{Discussion}

This pilot study addressed the pediatric practitioner's perspective on visit noncompliance to a cardiology subspecialty clinic. The results were similar to previous findings in that NS rates in a subspecialty clinic were relatively high despite visit prenotification. ${ }^{1-4}$ Unlike prior studies assessing adult primary practitioner's opinions about NS notification, most pediatric offices indicated that they likely would intervene if a NS was known. ${ }^{6}$ In contrast to our expected outcome, NS notification did not result in more successful encounters following a NS. Reasons were not clear. In some cases, the reason for the initial referral no longer may have existed, the family sought attention elsewhere, or the family relocated.

It was concerning that a majority of patients from both groups with either suspected or known congenital heart disease were not seen despite standard attempts by our practice to contact families. It was not unexpected that university NS rates were significantly higher compared to outreach as the university practice sees a primarily inner city population where socioeconomic factors may influence show rate. ${ }^{7}$ Intervention patients were seen sooner compared to the non-notification Control patients suggesting that NS notification, based upon survey 
results, may have played a role in both rates of and time to a successful encounter.

The most common reason patients miss appointments is forgetfulness. ${ }^{7-9}$ Other explanations include resolution of symptoms, frustration with healthcare, lower socio-economic class, inadequate insurance, inconvenience, and long wait times.

Appointment reminders and/or incentives (free parking) result in modest improvements in NS rates $(0-40 \%))^{10,11}$ Potential barriers to the success of these interventions may include lack of a permanent residence or continuous phone service. Exit interviews describing consequences of visit non-compliance such as referral cancellation or a cash penalty reduce subsequent NS's by approximately $5 \% .^{12}$ When patients schedule their own appointments, NS rates also improve. ${ }^{13}$ However, scheduling an appointment can be daunting in face of language barriers, anxiety about the appointment, or transportation issues. Same day (walk-in) appointments are also effective, but may not be practical for high volume practices. ${ }^{14}$

In contrast to the multitude of studies characterizing patient factors resulting in missed appointments, there are few studies evaluating how NSs are viewed and managed by the primary care team. ${ }^{15-18}$ Although data are limited, survey and focus group data of primary practices suggested that patient factors were perceived as the main determinant for visit non-compliance as opposed to any practice factors. ${ }^{17}$ Such attitudes by health care teams may correlate with design of interventions that implement consequences for NSs (penalty or fine) rather than implementing changes at a practice level. ${ }^{17}$ Indeed, such measures lead to a modest impact on NS rates but effects are incomplete. $^{12}$

Some physicians reported that confronting patients about NSs may compromise the doctor-patient relation- ship. ${ }^{17}$ These concerns require further investigation to overcome this perceived barrier to communication. Our data suggested many practitioners would take responsibility for mitigating a NS, but an equal amount of respondents suggested that the family holds some responsibility in keeping the appointment. Whereas most surveyed pediatric respondents would attempt to contact families following a NS, the majority also indicated referrals should be cancelled as a result of chronic nonattendance. Thus, perceptions regarding visit non-compliance remain complicated.

Contacting patients immediately following a missed subspecialty visit may be routine for many referral centers and may prompt patients to reschedule the appointment. ${ }^{4,15}$ Unfortunately, limited data suggested that a majority of patients may never be seen by the subspecialist. A phone survey of NS patients conducted by Ritzler et al. ${ }^{15}$ showed that only $47 \%$ of NS patients were seen elsewhere or had rescheduled. Indeed, some patients who were not seen may have reasonable justifications especially if they believed the visit was unnecessary or were dissatisfied with a previous encounter. ${ }^{16}$ Regardless, greater patient communication, even if as simple as visit reminders, leads to fewer NSs compared to no communication. ${ }^{18}$ To this end, by providing NS notification, the referring physician may play a critical role in investigating the NS, reassessing the need for the referral, and/or helping to eliminate barriers to keeping the visit.

Limitations. NS notification did not result in more patients being seen by the cardiologist following the initial missed visit as expected, but the Intervention group was seen sooner than non-notification controls. Because our study was small and only a minority of referring offices responded to the survey, we cannot be certain that the shorter time to follow-up was related to 
actions by the referring center in any or all instances. Preferred physician availability in our office and/or visit convenience also may have played a role. The referring office survey was non-validated, however, questions reflected content of prior publications. ${ }^{6}$ Finally, we cannot exclude the possibility that the use of the survey prior to the intervention influenced practitioner behavior and study outcome.

An important follow-up study would assess subsequent show rates between offices that act upon NS notification versus those that do not. Routine NS notification seems justified to improve physician-tophysician communication and enhance communication with the family. Advances in electronic medical record systems should

\section{References}

${ }^{1}$ Mugavero MJ, Lin HY, Willig JH, et al. Missed visits and mortality among patients establishing initial outpatient HIV treatment. Clin Infect Dis 2009; 48(2): 248256. PMID: 19072715.

${ }^{2}$ Fukushima H, Kato S, Kitano S, Yokoyama H, Hori S. Influence of dropout from medical follow-up on visual acuity prognosis in early-onset type 2 diabetes mellitus. Invest Ophthalmol Vis Sci 2004; 4:E-Abstract 4136.

${ }^{3}$ Chariatte V, Michaud PA, Berchtold A, Akré C, Suris JC. Missed appointments in an adolescent outpatient clinic: Descriptive analyses of consultations over 8 years. Swiss Med Wkly 2007; 137(47-48):677681. PMID: 18058276.

4 Mitchell AJ, Selmes T. Why don't patients attend their appointments? Maintaining engagement with psychiatric services. Adv Psychiatr Treat 2007; 13:423-434.

${ }^{5}$ Weiner M, Perkins AJ, Callahan CM. Errors in completion of referrals among older urban adults in ambulatory care. J Eval Clin Pract 2010; 16(1):76-81. PMID: 20367818. automate NS notification and minimize additional workloads for referral centers.

\section{Conclusion}

Although our study intervention of NS notification did not result in a greater number of successful encounters following a missed visit, referring centers seemed interested in obtaining NS notification and likely would contact the family to explore reasons behind the missed visit. NS notification may have led to earlier follow up compared to the non-notification group. Knowledge of a NS would help the referring practitioner reinforce the importance of a visit for patients judged to have the greatest risk for significant cardiovascular disease.

${ }^{6}$ Killaspy H, Banerjee S, King M, Lloyd M. Non-attendance at psychiatric outpatient clinics: Communication and implications for primary care. Br J Gen Pract 1999; 49(448):880-883. PMID: 10818652.

${ }^{7}$ Busnello RG, Melchior R, Faccin C, et al. Characteristics associated with the dropout of hypertensive patients followed up in an outpatient referral clinic. Arq Bras Cardiol 2001; 76(5):349-354. PMID: 11359183.

${ }^{8}$ Duarte MT, Cyrino AP, Cerqueira AT, Nemes MI, Iyda M. [Reasons for medical follow-up dropout among patients with arterial hypertension: The patient's perspective.] Cien Saude Colet 2010; 15(5):2603-2610. PMID: 20802892.

${ }^{9}$ King A, David D, Jones HS, O'Brien C. Factors affecting non-attendance in an ophthalmic outpatient department. J R Soc Med 1995; 88(2):88-90. PMID: 7769601.

${ }^{10}$ Stone CA, Palmer JH, Saxby PJ, Devaraj VS. Reducing non-attendance at outpatient clinics. J R Soc Med 1999; 92(3):114-118. PMID: 10396253. 
${ }^{11}$ Guy R, Hocking J, Wand H, Stott S, Ali $\mathrm{H}$, Kaldor J. How effective are short message service reminders at increasing clinic attendance? A meta-analysis and systematic review. Health Serv Res 2012; 47(2):614-632. PMID: 22091980.

${ }^{12}$ Guse CE, Richardson L, Carle M, Schmidt K. The effect of exit-interview patient education on no-show rates at a family practice residency clinic. J Am Board Fam Pract 2003; 16(5):399-404. PMID: 14645330.

${ }^{13}$ Versel N. Online reservations: Letting patients make their own appointments. amednews.com. March 22/29, 2004. Accessed at: http://api1.net/download/401.

${ }^{14}$ Cameron S, Sadler L, Lawson B. Adoption of open-access scheduling in an academic family practice. Can Fam Physician 2010; 56(9):906-911. PMID: 20841595.
${ }^{15}$ Carpenter PJ, Morrow GR, Del Gaudio AC, Ritzler BA. Who keeps the first outpatient appointment? Am J Psychiatry 1981; 138(1):102-105. PMID: 7446758.

${ }^{16}$ Zisook S, Hammond R, Jaffe K, Gammon E. Outpatient requests, initial sessions and attrition. Int J Psychiatry Med 1978-1979; 9(3-4):339-350. PMID: 757223.

${ }^{17}$ Hussain-Gambles M, Neal RD, Dempsey O, Lawlor DA, Hodgson J. Missed appointments in primary care: Questionnaire and focus group study of health professionals. Br J Gen Pract 2004; 54(499):108-113. PMID: 14965389.

${ }^{18}$ Kourany R, Garber J, Tornusciolo G. Improving first appointment attendance rates in child psychiatry outpatient clinics. J Am Acad Child Adolesc Psychiatry 1990; 29(4):657-660. PMID: 2387803.

Keywords: patient compliance, referral and consultation, pediatrics, cardiology 


\section{Appendix}

\section{Missed Cardiology Appointments: Survey}

1. Are you usually informed about missed subspecialty visits?

Yes $\quad$ No

2. How would you prefer to be notified of a missed visit by the referral center?

Letter Phone Other

3. When a missed visit is recognized, would you:
a. Call the referral center to reschedule
b. Ask the patient/family to call the referral center for a new appointment
c. Wait for the referral center to re-schedule to patient
d. Allow the family to decide whether or not to keep the appointment
e. Other

4. What would influence your decision whether to contact the family after a missed visit?

5. Who may be most influential in making sure missed appointment are eventually kept?
Referring physician Patient/family
Referral Center
Not sure

6. Should referral centers cancel consults after continued visit non-compliance $(>3)$ ?
Yes
No
Not sure 